\title{
Gli3 silencing enhances cyclopamine suppressive effects on ovarian cancer
}

This article was published in the following Dove Press journal:

OncoTargets and Therapy

30 October 2014

Number of times this article has been viewed

\section{Hai-Yan Liu}

Zheng Dong

Department of Gynecology, First Affiliated Hospital of Liaoning Medical College, Jinzhou, People's Republic of China
Correspondence: Hai-Yan Liu

Department of Gynecology, Department Unit 3, First Affiliated Hospital of Liaoning Medical College, Jinzhou I2100I, Liaoning province, People's Republic of China Email liuhaiyan_In@I63.com

\begin{abstract}
Ovarian cancer is a leading gynecological malignancy associated with high mortality. Hedgehog signaling has been found to be important for cell proliferation and tumor growth for multiple cancers, including ovarian cancer. The present study showed that the drug cyclopamine, which blocks the hedgehog signaling pathway, could reduce cancer cell growth and proliferation and induce cell apoptosis. In addition, the silencing of the glioma-associated oncogene (Gli)3, a downstream component of the hedgehog signaling pathway, could further enhance the antitumor effects of cyclopamine. Our results suggest that Gli3 may act as resistance to cyclopamine's effect on tumor growth. The combined treatment of cyclopamine application and Gli3 silencing therapy, therefore, may provide novel directions for clinical management of ovarian cancer.
\end{abstract}

Keywords: ovarian cancer, hedgehog, cyclopamine, proliferation, apoptosis, Gli3

\section{Introduction}

Ovarian cancer is a leading gynecological malignancy associated with high mortality. The management of ovarian cancer with pharmacological compounds is however still limited. Hedgehog signaling represents an important target in controlling cancer cell proliferation and progression, including that of ovarian cancer cells. ${ }^{1-5}$ For instance, the cyclopamine that blocks hedgehog signaling by antagonizing smoothened (smo) function has been employed to induce cancer cell apoptosis in different types of cancer cell lines, including those of prostate cancer, pancreatic cancer, ovarian cancer cells, and breast cancer. ${ }^{6-11}$

Downstream to smo protein, recent studies revealed the role of the glioma-associated oncogene (Gli) in the hedgehog signaling pathway. ${ }^{12-14}$ The Gli family includes several homologs such as Gli3, which has been proposed to be important in cancer development and tumor maintenance. ${ }^{15-17}$ Gli protein signaling has been recognized in the development of ovarian cancer, ${ }^{18}$ however, the exact role of Gli3 protein in hedgehog signaling in ovarian cancer proliferation and maintenance has not been fully investigated.

The present study examined the involvement of Gli3 signaling in the suppressive effects of cyclopamine on human ovarian cancer cell lines. The results suggest novel directions in targeting ovarian cancer at various molecular levels.

\section{Materials and methods} \section{Ethics statement}

The study was approved by the Liaoning Medical College Animal Research Committee, and all experimental procedures were carried out in accordance with the guidelines of medical research in Liaoning Medical College. 


\section{Cell culture}

The human ovarian SKOV3 cancer cell line was obtained from the Liaoning Medical College Cell Research Center and maintained in 10\% fetal bovine serum, antibiotics, and RPMI 1640 culture medium. Cyclopamine at $20 \mu \mathrm{M}$ was used to induce cell proliferation arrest and apoptosis as previously described. ${ }^{6}$

\section{Cell proliferation analysis}

The Premix WST-1 Cell Proliferation Assay (Takara Bio, Shiga, Japan) was employed for cell proliferation evaluation according to the manufacturer's instructions in order to determine the cell proliferation rates. The assays were repeated three times.

\section{Cell viability assay and apoptosis analysis}

The 3-(4,5-dimethylthiazol-2-yl)-2,5-diphenyltetrazolium bromide (MTT) assay was employed to examine cell viability, using a Millipore MTT kit (Billerica, MA, USA). The MTT assay was repeated five times.

To detect cell apoptosis in culture, the TUNEL kit (Roche, Basel, Switzerland) was used, and TUNEL-positive cells were counted using Hoechst counterstain for all the rest of the cells. The ratios of TUNEL positive to normal cells were calculated.

\section{Small interfering RNA for Gli3}

Gli3-specific small interfering RNAs (siRNAs) were obtained as previously described ${ }^{16}$ (siRNA-G1, 5'-UGA AUG GAA UGU UUC CGC GAC UGA A-3'; siRNA-G2, 5'-CCA UUG CAU AUG ACU UCC GCC UUA U-3'; $60 \mathrm{nM}$ ) (Shanghai Shengji Biotechnol, Shanghai, People's Republic of China). Lipofectamine 2000 reagent (Life Technologies, Carlsbad, CA, USA) was used for cell culture transfection. The transfection was performed 48 hours prior to the experiment.

\section{Xenograft}

SKOV3 cancer cells $\left(2 \times 10^{6}\right)$ in $200 \mu \mathrm{L}$ saline were injected into the intraperitoneal cavity of adult male nude mice 2 weeks prior to treatment to promote tumor growth. Mice were divided into four groups with 12 animals per group: control group received saline injection every 3 days, cyclopamine treatment group ( $25 \mathrm{mg} / \mathrm{kg}$ every 3 days), cyclopamine plus G1 siRNA group, and cyclopamine plus G2 siRNA group, also every 3 days. Cyclopamine was injected intraperitoneally while G1 and G2 were administered via the tail vein.
Mice were sacrificed at 4 weeks ( 6 animals in each group) and 7 weeks ( 6 animals in each group) after grafting of cancer cells, and tumor sizes were measured. Sections of the tumors were prepared for TUNEL staining, and the percentage of apoptotic cells was counted using a Zeiss microscope (Oberkochen, Germany).

\section{Statistics}

The data were represented as mean \pm standard deviation and analyzed with SPSS 17.0 software (IBM Corporation, Armonk, NY, USA). The results between different groups were analyzed by Student's $t$-test and ANOVA. $P<0.05$ was considered significantly different.

\section{Results \\ Cyclopamine suppresses cancer cell proliferation}

We found that the cyclopamine treatment significantly decreased cell proliferation after time lengths of 12, 24, and 48 hours (Figure 1).

\section{Cyclopamine decreases cell viability and induces cell apoptosis}

We further examined if cyclopamine decreased cell proliferation by the induction of cell apoptosis. Indeed, we found that accompanying the decrease in cell viability, TUNELpositive cells (apoptotic) increased in a time-dependent manner in cell culture (Figure 2). Given the clear effects of cyclopamine at 24 hours after treatment, we selected 24 hours as the time point for mechanistic investigations in the following studies.

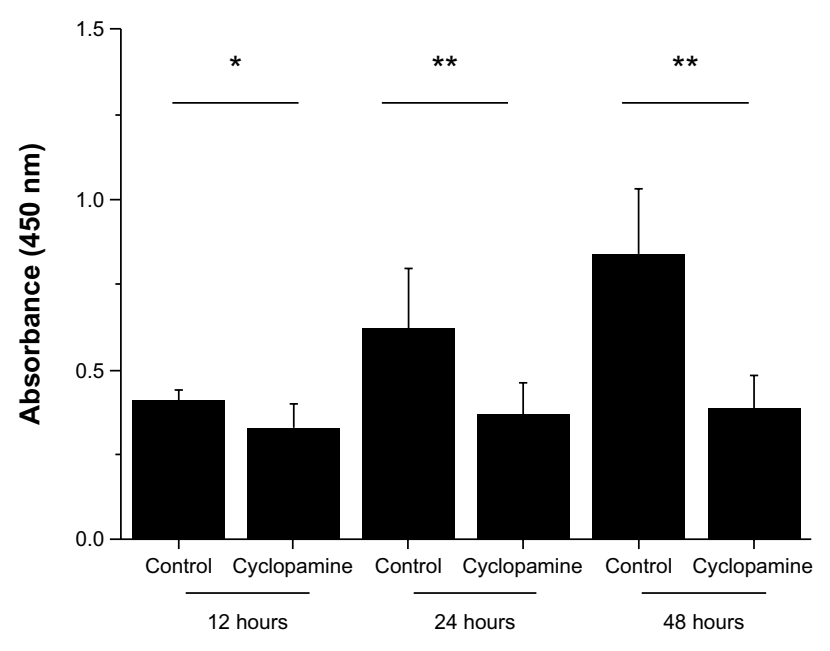

Figure I Cyclopamine suppresses cancer cell proliferation at 12-, 24-, and 48-hour time points.

Notes: Light absorbance at $450 \mathrm{~nm}$; *represents $P<0.05$; **represents $P<0.01$ compared to the control group. 


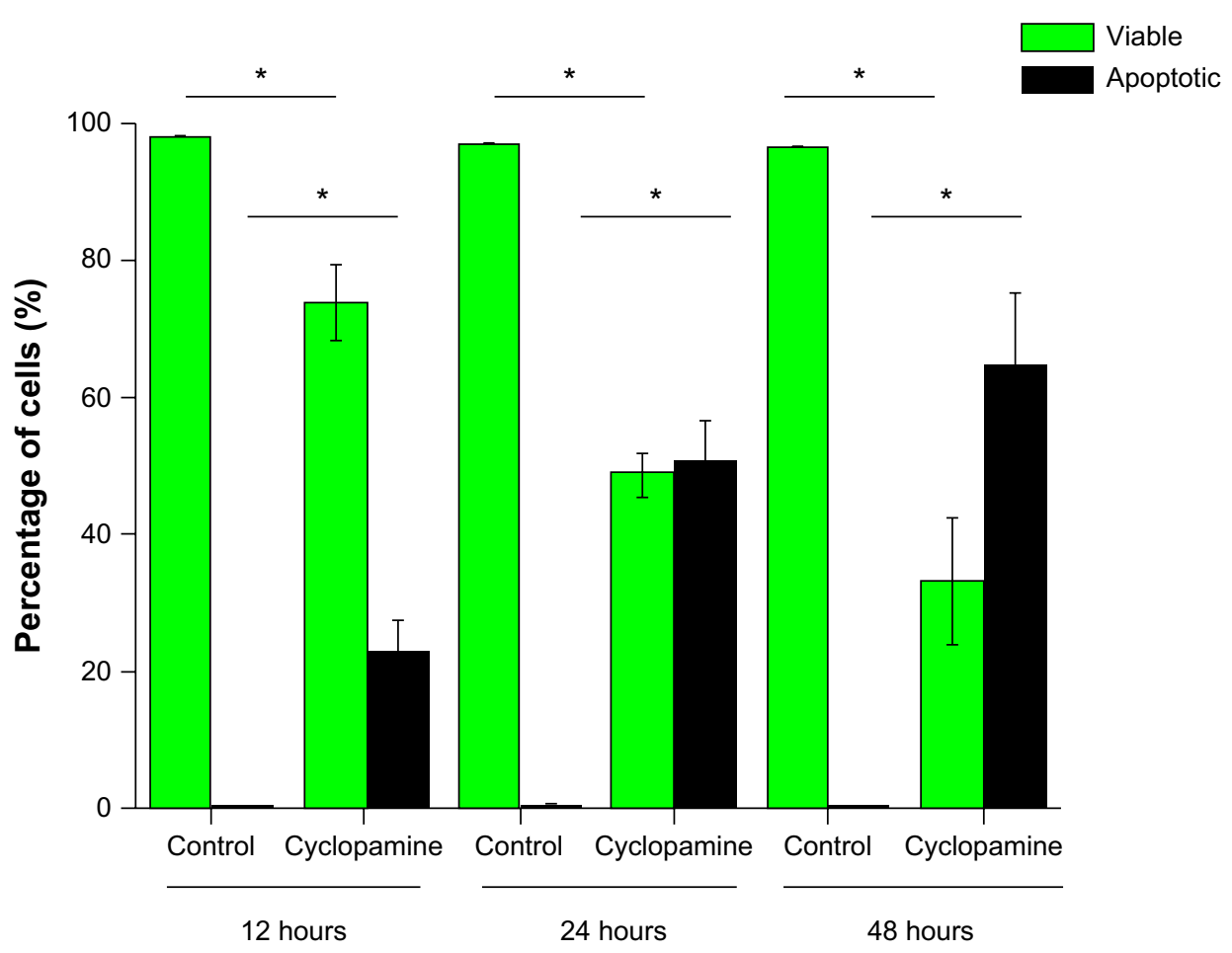

Figure 2 Cyclopamine decreases cell viability and induces cell apoptosis at 12-, 24-, and 48-hour time points. Note: *Represents $P<0.01$.

\section{Gli3 silencing further enhances cyclopamine effects}

We employed two siRNAs for Gli3 (siRNA-G1 and siRNA-G2) to silence the expression of Gli3. We confirmed that the two siRNAs did reduce the expression levels of Gli3 to less than $15 \%$ in our preliminary experiments. Interestingly, this silencing of Gli3 further enhanced the cyclopamine effects to suppress the cancer cell proliferation at the 24-hour time point (Figure 3). Gli3 silencing did not affect the baseline cell proliferation rate however (Figure 3).

We further examined the usefulness of Gli3 silencing on cyclopamine effects on cell viability and apoptosis at 24 hours. Gli3 silencing did not affect the baseline cell viability or apoptosis rate, yet both G1 and G2 siRNA treatment enhanced the cyclopamine-induced cell apoptosis of cultured cells (Figure 4).

\section{Gli3 silencing in vivo enhances the antitumor growth effect of cyclopamine}

In order to further validate the effects we observed on cultured cancer cells, we performed xenograft experiments and systematically infused siRNA-G1 and siRNA-G2 via the tail vein. We found that cyclopamine treatment could effectively induce tumor size decrease (Table 1) as well as cell apoptosis in the tumor tissue (Figure 5). Both effects were enhanced with G1 and G2 treatment (Figure 5).

\section{Discussion}

Ovarian cancer is a challenging malignancy for women's health. Recent studies highlighted the role of hedgehog sig-

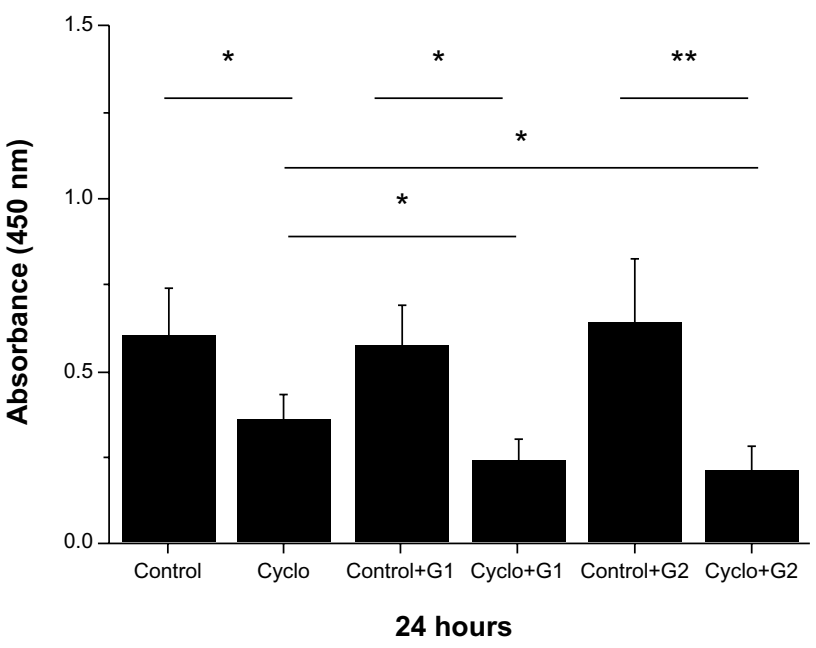

Figure 3 Gli3 silencing with two independent siRNAs further suppressed cancer cell proliferation.

Notes: Light absorbance at $450 \mathrm{~nm}$; *represents $P<0.05$; **represents $P<0.0$ I compared to the relevant group.

Abbreviations: Cyclo, cyclopamine; GI, siRNA-GI (5'-UGA AUG GAA UGU UUC CGC GAC UGA A-3'); G2, siRNA-G2 (5'-CCA UUG CAU AUG ACU UCC GCC UUA U-3'); siRNA, small interfering RNA. 


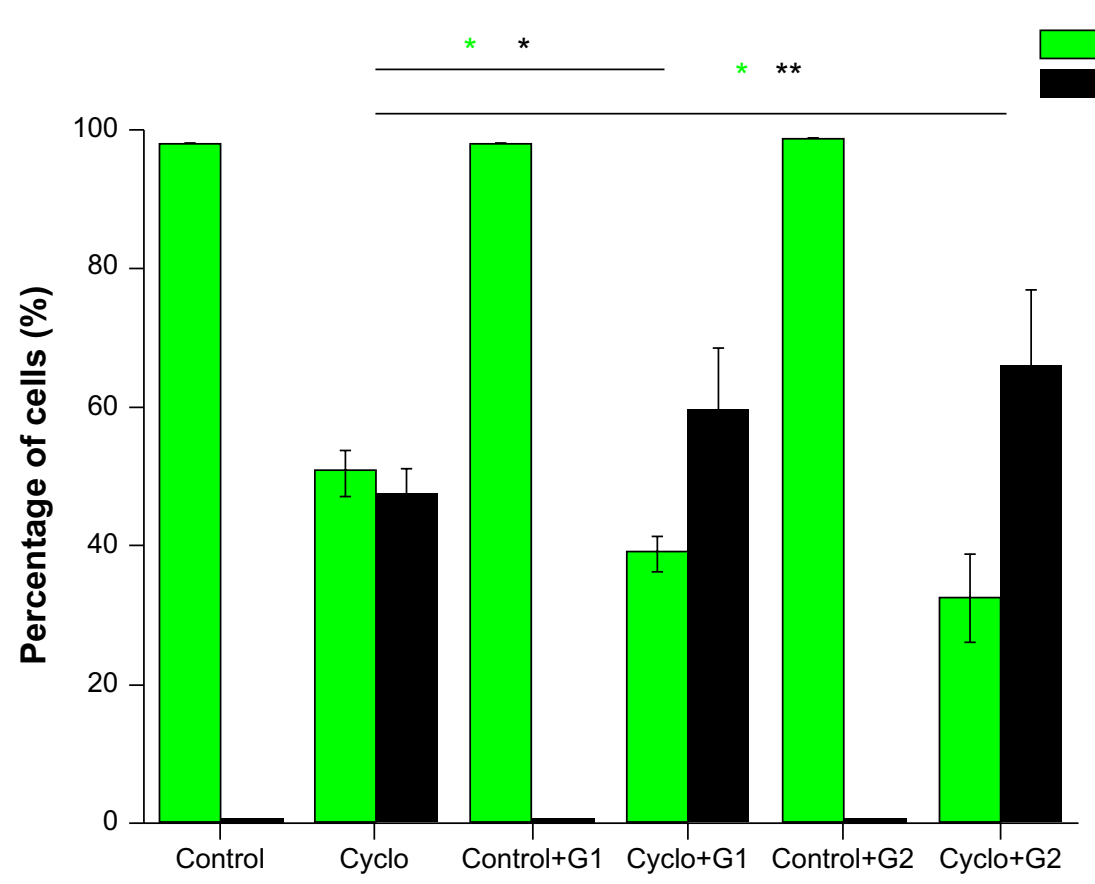

24 hours

Figure 4 Gli3 silencing with two independent siRNAs further decreased cell viability and induced cell apoptosis in addition to the cyclopamine effects at I2-, 24-, and 48hour time points.

Notes: *Represents $P<0.05$; **represents $P<0.01$ compared to the relevant group.

Abbreviations: Cyclo, cyclopamine; GI, siRNA-GI (5'-UGA AUG GAA UGU UUC CGC GAC UGA A-3'); G2, siRNA-G2 (5'-CCA UUG CAU AUG ACU UCC GCC UUA U-3'); siRNA, small interfering RNA.

naling in ovarian cancer development and progression. ${ }^{18,19,21}$ Cyclopamine has been applied for different diseases, with proven roles in antitumor growth as well as an inducer of cell apoptosis. ${ }^{1,2,20,21}$ The present study demonstrated that Gli3 may act to exert "resistance" downstream to the hedgehog signaling pathway, thereby partly antagonizing the effects of cyclopamine. The synergistic effect of cyclopamine application and Gli3 knockdown, therefore, represents a novel strategy for clinical management of ovarian cancer.

A previous study revealed that the differences in sensitivity to cyclopamine across different pancreatic cancer cell lines were mediated by Gli3 function. ${ }^{16}$ This is in line with the current results. It will be interesting to perform gain of

Table I Gli3 silencing in vivo enhances the antitumor growth effect of cyclopamine

\begin{tabular}{lllll}
\hline $\begin{array}{l}\text { Tumor } \\
\text { size }\end{array}$ & Control & Cyclopamine & Cyclo + GI & Cyclo + G2 \\
\hline 4 weeks & $645 \pm 114$ & $504 \pm 106^{*}$ & $320 \pm 76^{* * \ldots \#}$ & $339 \pm 82^{* *, \#}$ \\
7 weeks & $862 \pm 190$ & $622 \pm 127^{* *}$ & $394 \pm 93^{* * \ldots \#}$ & $427 \pm 115^{* *, \#}$ \\
\hline
\end{tabular}

Notes: Tumor size in $\mathrm{mm}^{3}$; *represents $P<0.05$; **represents $P<0.01$ compared to the control group; " represents $P<0.05$; ${ }^{\#}$ represents $P<0.01$ compared to the cyclopamine group.

Abbreviations: Cyclo, cyclopamine; GI, siRNA-GI (5'-UGA AUG GAA UGU UUC CGC GAC UGA A-3'); G2, siRNA-G2 (5'-CCA UUG CAU AUG ACU UCC GCC UUA U-3'); siRNA, small interfering RNA. function studies on Gli3 to see if this could promote tumor cell growth at the baseline level. Interestingly, in our study, Gli3 silencing did not cause much change in cell proliferation, cell viability, and apoptosis compared to the baseline level. It is possible that at baseline level, Gli3 is not actively involved in tumor growth; however, Gli3 is recruited when the

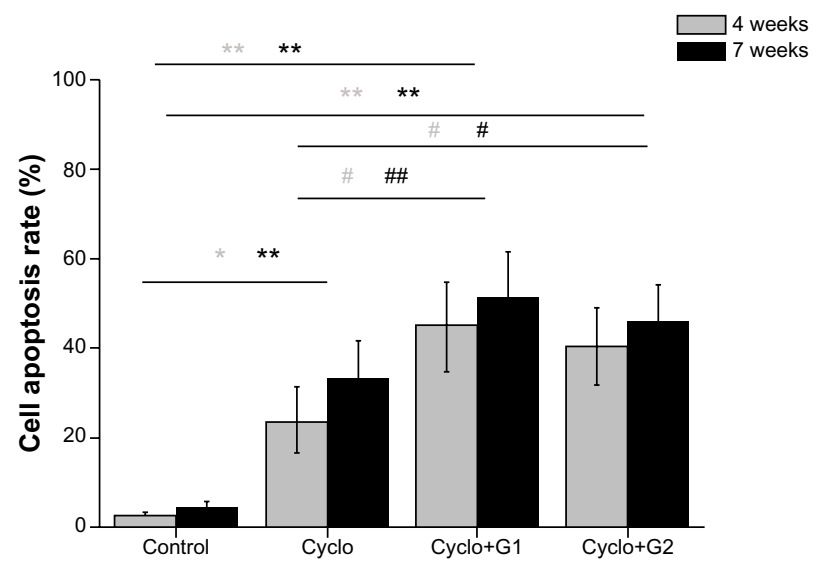

Figure $5 \mathrm{Gli} 3$ silencing in vivo increased the antitumor growth effect of cyclopamine through upregulation of cell apoptosis at both the 4-week and 7-week time points. Notes: *Represents $P<0.05$; **represents $P<0.01$ compared to the control group; "represents $P<0.05$; \#represents $P<0.01$ compared to the cyclopamine group. Abbreviations: Cyclo, cyclopamine; GI, siRNA-GI (5'-UGA AUG GAA UGU UUC CGC GAC UGA A-3'); G2, siRNA-G2 (5'-CCA UUG CAU AUG ACU UCC GCC UUA U-3'); siRNA, small interfering RNA. 
tumor cells were challenged with different chemotherapeutic drugs for instance.

The synergistic effects of cyclopamine and Gli3 silencing suggest that combined therapy would be more effective in targeting the hedgehog signaling pathway to manage ovarian cancer growth. There are numerous inhibitors for hedgehog signaling pathway being developed and tested in preclinical phases. Cyclopamine mainly acts on smo function. ${ }^{2,7}$ It will be important to find certain agents that target both Gli3 and smo function with a potentially high efficiency.

Other Gli3 proteins such as Gli1 have been found to be associated with ovarian cancer growth, invasion, and induced differentiation. ${ }^{12,22}$ In addition, cyclopamine or Gli1 silencing both suppressed ovarian cancer cell growth significantly. ${ }^{23}$ It will be important to investigate the potential functional redundancy between Gli1 and Gli3 in order to explore their interactions when ovarian cells are treated with cyclopamine.

\section{Acknowledgment}

The authors were supported by the Department of Gynecology at the First Affiliated Hospital of Liaoning Medical College.

\section{Disclosure}

The authors report no conflicts of interest in this work.

\section{References}

1. Szkandera J, Kiesslich T, Haybaeck J, Gerger A, Pichler M. Hedgehog signaling pathway in ovarian cancer. Int $J$ Mol Sci. 2013;14(1): 1179-1196.

2. Liu H, Gu D, Xie J. Clinical implications of hedgehog signaling pathway inhibitors. Chin J Cancer. 2011;30(1):13-26.

3. Chase DM, Mathur N, Tewari KS. Drug discovery in ovarian cancer. Recent Pat Anticancer Drug Discov. 2010;5(3):251-260.

4. Yang L, Xie G, Fan Q, Xie J. Activation of the hedgehog-signaling pathway in human cancer and the clinical implications. Oncogene. 2010;29(4):469-481.

5. Liao X, Siu MK, Au CW, et al. Aberrant activation of hedgehog signaling pathway contributes to endometrial carcinogenesis through beta-catenin. Mod Pathol. 2009;22(6):839-847.
6. Che J, Zhang FZ, Zhao CQ, Hu XD, Fan SJ. Cyclopamine is a novel Hedgehog signaling inhibitor with significant anti-proliferative, antiinvasive and anti-estrogenic potency in human breast cancer cells. Oncol Lett. 2013;5(4):1417-1421.

7. Katano M. Hedgehog signaling pathway as a therapeutic target in breast cancer. Cancer Lett. 2005;227(2):99-104.

8. Sirab N, Terry S, Giton F, et al. Androgens regulate Hedgehog signalling and proliferation in androgen-dependent prostate cells. Int J Cancer. 2012;131(6):1297-1306.

9. Shaw G, Price AM, Ktori E, et al. Hedgehog signalling in androgen independent prostate cancer. Eur Urol. 2008;54(6):1333-1343.

10. Kelleher FC, McDermott R. Aberrations and therapeutics involving the developmental pathway Hedgehog in pancreatic cancer. Vitam Horm. 2012;88:355-378.

11. McCann CK, Growdon WB, Kulkarni-Datar K, et al. Inhibition of hedgehog signaling antagonizes serous ovarian cancer growth in a primary xenograft model. PLoS One. 2011;6(11):e28077.

12. Ciucci A, De Stefano I, Vellone VG, et al. Expression of the gliomaassociated oncogene homolog 1 (gli1) in advanced serous ovarian cancer is associated with unfavorable overall survival. PLoS One. 2013;8(3):e60145

13. Li H, Lui N, Cheng T, et al. Gli as a novel therapeutic target in malignant pleural mesothelioma. PLoS One. 2013;8(3):e57346.

14. Gao J, Khan AA, Shimokawa T, et al. A feedback regulation between Kindlin-2 and GLI1 in prostate cancer cells. FEBS Lett. 2013;587(6); 631-638.

15. Mazumdar T, DeVecchio J, Agyeman A, Shi T, Houghton JA. The GLI genes as the molecular switch in disrupting Hedgehog signaling in colon cancer. Oncotarget. 2011;2(8):638-645.

16. Steg A, Amm HM, Novak Z, Frost AR, Johnson MR. Gli3 mediates cell survival and sensitivity to cyclopamine in pancreatic cancer. Cancer Biol Ther. 2010;10(9):893-902.

17. Katoh Y, Katoh M. Hedgehog signaling pathway and gastric cancer. Cancer Biol Ther. 2005;4(10):1050-1054.

18. Chen X, Horiuchi A, Kikuchi N, et al. Hedgehog signal pathway is activated in ovarian carcinomas, correlating with cell proliferation: it's inhibition leads to growth suppression and apoptosis. Cancer Sci. 2007;98(1):68-76.

19. Bhattacharya R, Nicoloso M, Arvizo R, et al. MiR-15a and MiR-16 control Bmi-1 expression in ovarian cancer. Cancer Res. 2009;69(23): 9090-9095.

20. Yang L, Su X, Xie J. Activation of Hedgehog pathway in gastrointestinal cancers. Vitam Horm. 2012;88:461-472.

21. Campos SM, Ghosh S. A current review of targeted therapeutics for ovarian cancer. J Oncol. 2010;2010:149362.

22. Kudo K, Gavin E, Das S, Amable L, Shevde LA, Reed E. Inhibition of Gli1 results in altered c-Jun activation, inhibition of cisplatin-induced upregulation of ERCC1, XPD and XRCC1, and inhibition of platinumDNA adduct repair. Oncogene. 2012;31(44):4718-4724.

23. Kandala PK, Srivastava SK. Diindolylmethane-mediated Gli1 protein suppression induces anoikis in ovarian cancer cells in vitro and blocks tumor formation ability in vivo. J Biol Chem. 2012;287(34): 28745-28754.
OncoTargets and Therapy

\section{Publish your work in this journal}

OncoTargets and Therapy is an international, peer-reviewed, open access journal focusing on the pathological basis of all cancers, potential targets for therapy and treatment protocols employed to improve the management of cancer patients. The journal also focuses on the impact of management programs and new therapeutic agents and protocols on

\section{Dovepress}

patient perspectives such as quality of life, adherence and satisfaction. The manuscript management system is completely online and includes a very quick and fair peer-review system, which is all easy to use. Visit http://www.dovepress.com/testimonials.php to read real quotes from published authors. 